\title{
EFECTOS DE Eisenia foetida y Eichhornia crassipes EN LA REMOCIÓN DE MATERIA ORGÁNICA, NUTRIENTES Y COLIFORMES EN EFLUENTES DOMÉSTICOS
}

\section{EFFECTS OF Eisenia foetida AND Eichhornia crassipes IN THE REMOVAL OF ORGANIC MATTER, NUTRIENTS AND COLIFORMS IN DOMESTIC WASTEWATER}

\author{
Lissette Vizcaíno Mendoza ${ }^{1}$, Natalia Fuentes Molina ${ }^{2}$
}

${ }^{1}$ Ing. Medio Ambiente. Universidad de La Guajira. Carrera 4 \# 14 $4^{\mathrm{a}}$-79, Riohacha-La Guajira, Colombia, e-mail: lvizcaino@ uniguajira.edu.co; ${ }^{2}$ M.Sc. Ciencias Ambientales. Universidad de La Guajira. Carrera 9 N 3 Sur - 20, San Juan del Cesar, La Guajira, Colombia, e-mail: nnfuentes@uniguajira.edu.co

Rev. U.D.C.A Act. \& Div. Cient. 19(1): 189-198, Enero-Junio, 2016

\section{RESUMEN}

Con el objeto de conocer la capacidad depuradora de un sistema biológico potencialmente aplicable como alternativa para la reducción de la carga contaminante de efluentes residuales domésticos del municipio de San Juan del Cesar, se evaluó, a escala de laboratorio, el efecto del tratamiento con Eisenia foetida y Eichhornia crassipes, sobre la remoción de materia orgánica, de nutrientes y de patógenos. Durante un mes, se aplicaron tres tratamientos: T1: E. foetida (biofiltro dinámico aerobio de flujo vertical); T2: $E$. crassipes (sistema de flujo horizontal con plantas flotantes) y T3: E. foetida + $E$. crassipes (sistema en serie), empleando tiempos de retención hidráulica de 24 h y caudales de $0,125 \mathrm{~L} / \mathrm{min}$ (E. foetida) y $0,020 \mathrm{~L} / \mathrm{min}$ (E. crassipes). Se determinaron las concentraciones de sólidos totales (ST), sólidos suspendidos totales (SST), turbidez, Demanda Bioquímica de Oxígeno (DBO5), Demanda Química de Oxígeno (DQO), Nitrógeno Total, fosfatos, coliformes totales y Escherichia coli, en 30 muestras analizadas (10 para cada sistema). T1 fue más eficiente en la remoción de DQO 69,2\%, T2 eliminó el 100\% de SST, mientras que T3 reportó las mejores eficiencias de remoción, para la demás variables analizadas. Existen diferencias significativas $p<0,05$ en la remoción de materia orgánica, nutrientes y coliformes, en los tratamientos aplicados. La matriz de correlación de Pearson indica que existe relación directa $p \leq 0,01$ entre la remoción de ST y la disminución de las concentraciones de DQO, coliformes totales y E. coli. Finalmente, la prueba de HDS Tukey confirmó que T3, excepto para DBO, resultó ser más eficientes en el tratamiento, las variables analizadas $\mathrm{p}<0,05$.

Palabras clave: DBO, DQO, humedales construidos, lombrifiltro, tratamiento biológico.
SUMMARY

In order to determine the purifying capacity of a potentially applicable biological system as an alternative for reducing the pollution load of domestic sewage effluent in the municipality of San Juan del Cesar, under laboratory scale the effect of treatment with Eisenia foetida and Eichhornia crassipes in the removal of organic matter, nutrients and pathogens was evaluated. During one month, three treatments were applied; T1: E. foetida (dynamic aerobic biofilter vertical flow), T2: $E$. crassipes (horizontal flow system with floating plants) and T3: E. foetida $+E$. crassipes (series system); using hydraulic retention times of $24 \mathrm{~h}$ and flow of $0,125 \mathrm{~L} / \mathrm{min}$ ( $E$. foetida) and $0,020 \mathrm{~L} / \mathrm{min}$ (E. crassipes). Concentration of total solids, total suspended solids (TSS), turbidity, biochemical oxygen demand (BOD5), chemical oxygen demand (COD), total nitrogen, phosphate, total coliforms and Escherichia coli in 30 analyzed samples (10 for each system) were determined. T1 was more efficient in the removal of COD $69.2 \%$; T2 eliminated $100 \%$ of TSS, while T3 reported the best removal efficiencies for the other variables analyzed. There are significant differences $p<0.05$ in the removal of organic matter, nutrients and coliforms by the treatments applied. The Pearson correlation matrix indicates that there is direct relationship between removal $p \leq 0.01 \mathrm{ST}$ and decreased concentrations of COD, total coliforms and E. coli. Finally Tukey HDS test confirmed that T3 except for BOD were the most efficient in the treatment of all the variables analyzed $p<0.05$.

Key words: BOD, COD, constructed wetlands, earthwormfilters, biological treatment. 


\section{INTRODUCCIÓN}

La contaminación de las aguas constituye un problema ambiental, generado como consecuencia de la descarga directa o parcialmente tratada de efluentes residuales, producto de actividades industriales y el crecimiento poblacional (Salgado et al. 2012). Estos, al mezclarse con cuerpos, los receptores generan un incremento en las concentraciones de materia orgánica, nutrimentos, compuestos tóxicos y microorganismos indeseables (Luna \& Ramírez, 2004).

En América Latina, las diferencias marcadas en los aspectos socioeconómicos, ambientales y tecnológicos reducen el acceso a sistemas o instalaciones mínimas, adecuadas para la depuración de los efluentes residuales, especialmente, en las zonas rurales (Montoya et al. 2010).

En este sentido, los sistemas biológicos convencionales, como lagunas de estabilización (Correa et al. 2012), tanques sépticos, filtros anaerobios de flujo ascendente (Seghezzo et al. 1998; Madera et al. 2005), en los que la remoción se da principalmente por la acción de microorganismos, se presentan como las principales alternativas de tratamiento; sin embargo, en la actualidad, los sistemas basados en el uso de plantas acuáticas (Romero et al. 2009; Rodríguez et al. 2010; Correa et al. 2015) y de lombrices (Ramón et al. 2015), sobresalen como una opción viable, con bajos costos de operación y simplicidad en el manejo tecnológico.

Los sistemas con plantas flotantes utilizan una cobertura de plantas, como la Eichhornia crassipes, cuyas características principales son la hiperacumulación de sustancias tóxicas (Peña et al. 2013) y transformación de compuestos, caracterizados por parámetros, como las demanda química de oxígeno y demanda bioquímica de oxígeno (DQO y DBO5), sólidos suspendidos totales (SST) y nutrientes (Martelo \& Lara, 2012). Los contaminantes en los sistemas con macrófitas son removidos por una variedad compleja de procesos biológicos, físicos y químicos, incluyendo sedimentación, filtración, adsorción en el suelo, degradación microbiológica, nitrificación y denitrificación, decaimiento de patógenos y metabolismo de las plantas (Peña et al. 2003).

Los biofiltros dinámicos aerobios de flujo vertical basados en el uso de lombrices, como Eisenia foetida, tienen alta eficiencia en la remoción de materia orgánica y organismos patógenos, por sus características físicas o estructurales (Ramón et al. 2015). Se diseñan con funcionamiento intermitente, por lo que operan con cargas superiores que los horizontales. La aplicación intermitente del agua residual y el drenaje vertical en el lecho permiten que las reacciones aeróbicas se produzcan con rapidez, permitiendo una mayor oxigenación del líquido (Pérez et al. 2015).
El presente estudio buscó determinar y comparar la eficiencia de remoción de materia orgánica, nutrientes, coliformes totales y $E$. coli de la macrofita $E$. crassipes, plantada en un sistema de flujo horizontal con plantas flotantes y la lombriz roja californiana $E$. foetida, sembrada en un biofiltro dinámico aerobio de flujo vertical, con lechos de aserrín y grava, empleando un caudal de agua residual, proveniente del pozo de inspección, ubicado a la entrada de la laguna de estabilización del municipio de San Juan del Cesar, sur del departamento de La Guajira.

\section{MATERIALES Y MÉTODOS}

La investigación, se desarrolló aplicando dos tratamientos individuales T1 y T2, empleando un biofiltro dinámico aerobio de flujo vertical, para las pruebas con E. foetida y un sistema de flujo horizontal con plantas flotantes, para los ensayos con E. crassipes. Un tercer tratamiento T3 consistió en analizar la capacidad combinada de las dos especies, convirtiendo las unidades en un sistema en serie biofiltro flujo vertical (lombrifiltro) - biofiltro flujo horizontal (sistema con plantas flotantes). En total, se analizaron 30 muestras, 10 para cada tratamiento; en la figura 1 , se muestran los esquemas para cada sistema de tratamiento empleado.

El lombrifiltro, se construyó con láminas de vidrio de $1 \mathrm{~m}$ de altura y 0,5m de ancho. El sistema, se diseñó con base en la metodología citada por Ramón et al. (2015), ubicando -en la parte inferior-, un lecho de soporte de $30 \mathrm{~cm}$, constituido por $15 \mathrm{~cm}$ de grava gruesa $(\phi=5 \mathrm{~cm})$ y $15 \mathrm{~cm}$ de grava fina; en la parte superior, se dispuso una capa de aserrín de $20 \mathrm{~cm}$, mezclada con $5 \mathrm{~kg}$ de E. foetida. La entrada del efluente al sistema, se hizo por la parte superior, a través de aspersión (Parra \& Chiang, 2014), empleando tubería de PVC de $1 / 2$ pulgada de diámetro, perforada con orificios de $5 \mathrm{~mm}$ de diámetro, cada $3 \mathrm{~cm}$. Los ensayos, se realizaron empleando un tiempo de retención hidráulica de $24 \mathrm{~h}$ y un caudal de $0,125 \mathrm{~L} / \mathrm{min}$, proveniente de un tanque de almacenamiento de 250L, el cual, actuó como un decantador primario.

El humedal artificial de flujo libre, se diseñó con una longitud de $0,9 \mathrm{~m}$, un diámetro de $0,60 \mathrm{~m}$ y una profundidad de 0,40m; un caudal de alimentación de 0,020L/min y un tiempo de retención $24 \mathrm{~h}$, de acuerdo con lo recomendado por Rodríguez et al. (2010). Los ejemplares de E. crassipes recolectados en el tramo del Río Ranchería, que bordea las instalaciones de la Universidad de La Guajira, Sede Fonseca, se sembraron con una densidad de seis plantas por metro cuadrado, que se aclimataron durante un mes, con agua residual del sistema de alcantarillado del municipio de San Juan del Cesar, La Guajira, a temperatura promedio de $28,3^{\circ} \mathrm{C}$. 


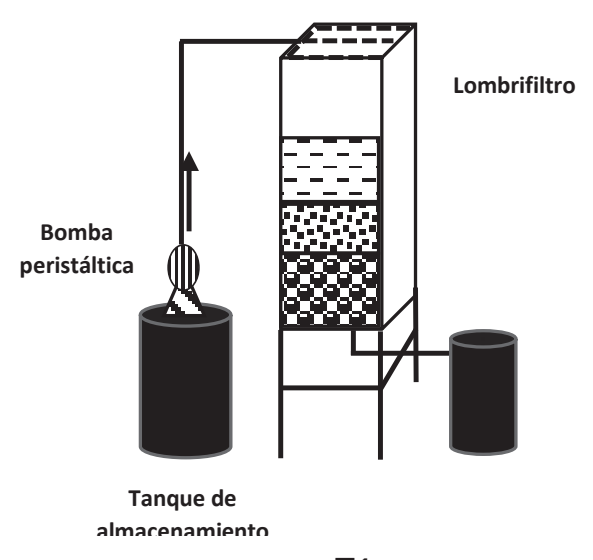

T1

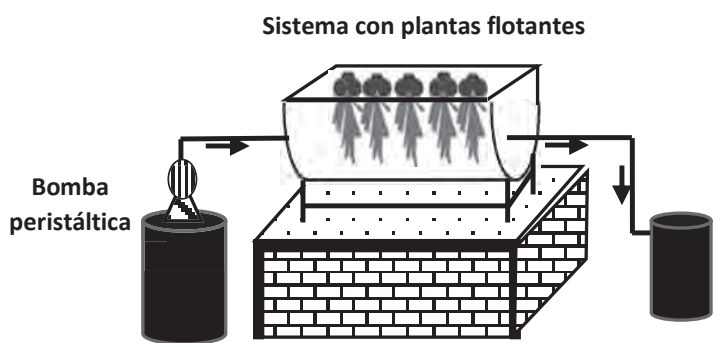

Tanque de almacenamiento

T2

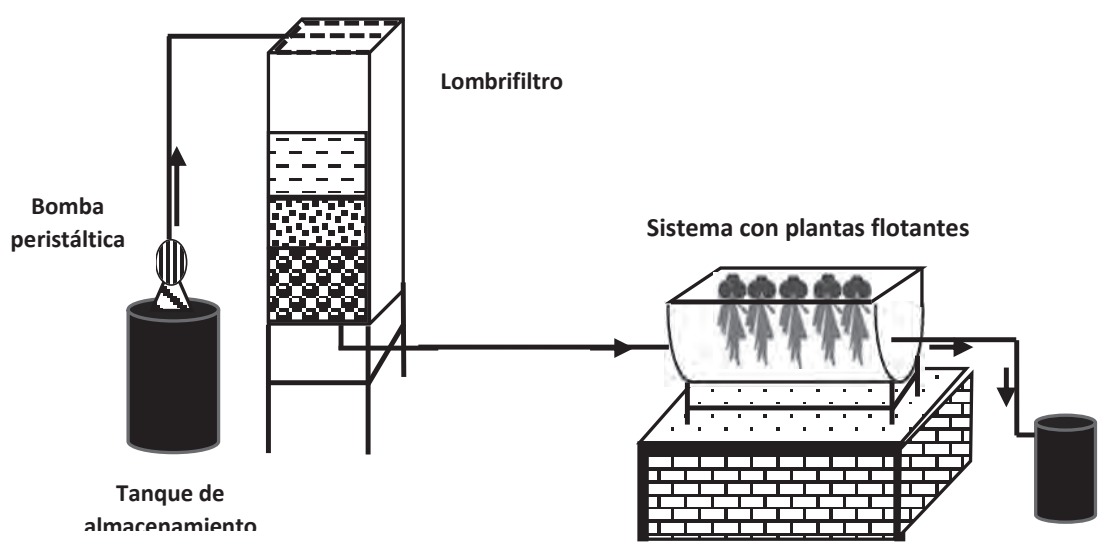

T3

Figura 1. Sistemas de tratamiento. T1) Biofiltro dinámico aerobio de flujo vertical con lecho de E. foetida; T2) Sistema con plantas flotantes de flujo horizontal, para tratamiento con E. crassipes; T3) sistema en serie para tratamiento combinado.

Los experimentos, se realizaron durante el periodo comprendido entre el 15 de marzo al 30 de abril de 2015, tiempo en el que se analizó el efecto de cada tratamiento, sobre las variables objeto de estudio: materia orgánica, medida como Sólidos Totales (ST), Sólidos Suspendidos Totales (SST), Turbidez, Demanda Bioquímica de Oxígeno (DQO) y Demanda Química de Oxígeno (DQO), nutrientes expresados en términos de las concentraciones de Nitrógeno Total y Fosfatos; patógenos, medidos como coliformes totales y $E$. coli; temperatura, Oxígeno Disuelto (OD) y $\mathrm{pH}$. Las variables, se analizaron de acuerdo con los métodos estándar (APHAAWWA-WPCF, 2005). El comportamiento de las concentraciones determinadas para cada una de las muestras tratadas será analizado, empleando diagramas de cajas y bigotes.

Análisis estadístico: Empleando el programa IBM SPSS Statistics Ver. 22, se realizó un análisis de homogeneidad de las varianzas Test de Levine, una prueba de distribución normal de los residuos Test de Kolmogorov-Smirnov y un estudio de correlación de Pearson, entre los parámetros fisicoquímicos y microbiológicos, para establecer posibles relaciones entre ellos, considerando un $p<0,05$. Un análisis de varianza (ANOVA) de una vía, a fin de determinar posibles diferencias estadísticas en los parámetros fisicoquímicos y microbiológicos, considerando el tipo de efluente, según el tratamiento aplicado y una prueba de diferencias honestamente significativas de Tukey (Marín et al. 2015).

\section{RESULTADOS Y DISCUSIÓN}

En la tabla 1, se presentan las concentraciones promedio de materia orgánica (ST, SST, Turbidez, $\mathrm{DBO}_{5}$ y DQO), nutrientes (NT y PT) y patógenos (coliformes totales y E. coli), porcentajes de remoción logrados por cada tratamiento, así como los resultados de las pruebas de homocedasticidad (Levine), normalidad (Kolmogorov - Smirnov), ANOVA y la prueba HDS de tukey. 
Tabla 1. Porcentajes de remoción de materia orgánica, de nutrientes y de microorganismos patógenos y análisis estadístico.

\begin{tabular}{|c|c|c|c|c|c|c|c|c|c|c|c|}
\hline \multirow{4}{*}{\multicolumn{2}{|c|}{ Tratamiento }} & \multicolumn{10}{|c|}{ Porcentaje de remoción } \\
\hline & & \multicolumn{5}{|c|}{ MATERIA ORGÁNICA } & & \multicolumn{2}{|c|}{ NUTRIENTES } & \multicolumn{2}{|c|}{ PATÓGENOS } \\
\hline & & ST & SST & Turb. & $\mathrm{DBO}_{5}$ & DQO & OD & $N$ total & Fosfatos & C. totales & E. coli \\
\hline & & $\mathrm{mg} / \mathrm{L}$ & $\mathrm{mg} / \mathrm{L}$ & UNT & $\mathrm{mg} / \mathrm{L}$ & $\mathrm{mg} / \mathrm{L}$ & $\mathrm{mg} / \mathrm{L}$ & $\mathrm{mg} / \mathrm{L}$ & $\mathrm{mg} / \mathrm{L}$ & $\mathrm{NMP} / 100 \mathrm{~mL}$ & $\mathrm{NMP} / 100 \mathrm{~mL}$ \\
\hline \multicolumn{2}{|c|}{ T1 } & 52,8 & 97,5 & 81,3 & 70,3 & 69,2 & 3,17 & 73,6 & 44,6 & 89,5 & 85,6 \\
\hline \multicolumn{2}{|c|}{$\mathrm{T} 2$} & 64,2 & 100 & 81,8 & 83,7 & 63,9 & 2,21 & 66,6 & 20 & 94,5 & 94,1 \\
\hline \multicolumn{2}{|c|}{ T3 } & 83 & 100 & 96 & 91 & 64 & 3,09 & 79 & 48 & 99 & 99 \\
\hline \multicolumn{2}{|c|}{$\begin{array}{l}\text { Concentración } \\
\text { inicial }\end{array}$} & 549 & 384 & 243 & 135 & 228 & 0,98 & 42,6 & 11,6 & $5,39 \times 10^{7}$ & $3,57 \times 10^{7}$ \\
\hline \multicolumn{12}{|c|}{ Kolmogorov - Smirnov } \\
\hline \multicolumn{2}{|c|}{ K-S } & 187 & ,362 & 223 & , 132 & ,122 &, 121 & ,192 & ,148 & ,172 & 203 \\
\hline \multicolumn{2}{|c|}{$\mathrm{p}$} & ,009 & , 000 &, 001 & , 189 & ,200 & ,200 & ,006 & 092 & ,024 & ,003 \\
\hline \multicolumn{12}{|c|}{ Levine } \\
\hline \multicolumn{2}{|c|}{ Levine } & 87,89 & & 1,140 & 3,290 & ,190 & 2,413 & 3,418 & 2,243 & 7,958 & 6,017 \\
\hline \multicolumn{2}{|c|}{$\mathrm{p}$} & ,000 & ,000 & ,295 & ,080 & ,666 & ,132 & ,075 & ,145 & ,009 & ,021 \\
\hline \multicolumn{12}{|c|}{ ANOVA } \\
\hline \multicolumn{2}{|c|}{$\mathrm{F}$} & 41,58 & 8,123 & 1,681 & 15,808 & , 154 & 2,413 & 1,881 & ,797 & 17,210 & 18,273 \\
\hline \multicolumn{2}{|c|}{$\mathrm{p}$} & ,000 & ,008 & ,205 & ,000 & ,698 & ,003 & ,181 & ,380 & ,000 & ,000 \\
\hline \multicolumn{12}{|c|}{ HDS Tukey } \\
\hline \multicolumn{12}{|c|}{ Subconjunto } \\
\hline \multirow{3}{*}{1} & $\mathrm{~T} 1$ & 92,6 & 9,5 & & & 74,85 & & 11,26 & 6,43 & & \\
\hline & $\mathrm{T} 2$ & & & & 21,95 & 81,33 & 2,21 & & 9,29 & 2971000 & 2100100 \\
\hline & T3 & & 8,3 & & 11,79 & 82,08 & 2,46 & 9,02 & 5,99 & 778000 & 356780 \\
\hline \multirow{2}{*}{2} & $\mathrm{~T} 1$ & & & 39,28 & & & & & & & \\
\hline & $\mathrm{T} 2$ & & & 42,94 & & & & & & & \\
\hline \multicolumn{2}{|c|}{$\mathrm{p}$} & 1,000 & 0,917 & 0,931 & 0,284 & 0,934 & 0,684 & 0,599 & 0,08 & 0,086 & 0,22 \\
\hline
\end{tabular}

T1: E. foetida; T2: E. crassipes; T3: E. foetida + E. crassipes. Concentraciones iniciales ST, SST, DBO5, DBQ, N total y Fosfatos en mg/l, Turbiedad en UNT y Coliformes en NMP/100mL.

Las características iniciales del efluente doméstico sin tratamiento corresponden a un agua residual de concentración media, según lo reportado en la literatura (Metcalf \& Eddy, 1995). De manera similar, se comportaron los valores de temperatura, de $\mathrm{pH}$ y de oxígeno disuelto del agua, en la entrada de los sistemas, reportándose valores promedio de $30,4^{\circ} \mathrm{C}\left( \pm 3,04^{\circ} \mathrm{C}\right), \mathrm{pH} 7,2$ ( $\pm 2,17$ unidades) y $0,98 \mathrm{mg} \mathrm{O}_{2} / \mathrm{L}$, respectivamente.

La prueba de Kolgomorov - Smirnov determinó que los valores de $\mathrm{DBO}_{5}(\mathrm{k}-\mathrm{s}=0,132 ; \mathrm{p}=0,189), \mathrm{DQO}(\mathrm{k}-\mathrm{s}=0,122 ; \mathrm{p}=0,200)$ y $\mathrm{P}(\mathrm{k}-\mathrm{s}=0,121 ; \mathrm{p}=0,200)$ provienen de una distribución normal ( $p>0,05)$. Los valores críticos del estadístico de Levene, para ST $(L=87,89 ; p=0,000)$, SST $(L=131,947 ; p=0,000)$, coliformes totales $(\mathrm{L}=7,958 ; \mathrm{p}=0,000)$, E. coli $(\mathrm{L}=6,017 \mathrm{y}$ $p=0,021$ ) son menores que 0,05 , con lo cual, se concluye que, en los tratamientos aplicados, las varianzas de dichas variables no son iguales; para las demás variables analizadas, la diferencia de varianzas muestrales no es significativa.

Los resultados del análisis de varianza (ANOVA) indican que no existen diferencias estadísticas entre los tres tratamientos aplicados para la remoción de Turbiedad $(p=0,205), D Q O$ $(p=0,698)$, NT $(p=0,181)$ y PT $(p=0,380)$; para el resto de las variables, se encontró una diferencia con $\mathrm{p} \leq 0,01$.

El análisis de correlación de Pearson (Tabla 2) indica que existe relación altamente significativa entre la remoción de ST y la disminución de las concentraciones de DBO, coliformes totales y E. coli, así como entre los niveles de DQO y la eliminación de fosfatos y nitrógeno total. 
Tabla 2. Matriz de correlación de Pearson entre las variables físicas, químicas y microbiológicas analizadas.

\begin{tabular}{|l|c|c|c|c|c|c|c|}
\hline & TURB. & DBO & DQO & N TOTAL & FOSFATOS & C. TOTALES & E. coli \\
\hline ST &, $461^{*}$ &, $651^{* *}$ & $-0,002$ & 0,261 & 0,128 &, $626^{* *}$ &, $642^{* *}$ \\
\hline TURB. & &, $413^{*}$ & $-0,006$ & 0,3 & 0,215 & 0,314 & 0,275 \\
\hline DBO & & & 0,207 & 0,276 & 0,025 &, $521^{* *}$ &, $521^{* *}$ \\
\hline DQO & & & &, $446^{*}$ &, $569^{* *}$ & 0,081 & $-0,027$ \\
\hline N TOTAL & & & & &, $563^{* *}$ & 0,208 & 0,011 \\
\hline FOSFATOS & & & & & & 0,104 & $-0,108$ \\
\hline C. TOTALES & & & & & & &, $570^{* *}$ \\
\hline
\end{tabular}

*. Significativo $(p \leq 0,05)$ (bilateral). **.Altamente significativo $(p \leq 0,01)$.

Los efluentes tratados con T1 y T3 reportaron el mayor incremento de oxígeno disuelto, equivalente al $210 \%$, mientras que con T2, se alcanzó una concentración final de $2,21 \mathrm{mgO}_{2} / \mathrm{L}$, lo cual, equivale a un incremento del $120 \%$; sin embargo, existe una mayor similitud entre las medias obtenidas en los tratamientos T2 y T3, con un nivel de significancia $\mathrm{p}=0,684$ (Tabla 1).

Los SST, se eliminaron en un $97,5 \%$, con $\mathrm{T} 1$ y $100 \%$, en T2 y T3, reportándose un comportamiento más homogéneo de los valores medios, obtenidos en T1 $(t=8,300)$ y T3 $(9,500)$, para $p=0,917$. En cuanto a los ST, que lograron ser removidos en un $83 \%$ por $\mathrm{T} 3$, los tratamientos no mostraron similitud entre las concentraciones medias finales obtenidas $(p=1,000)$.

T1 y T2 lograron remover la turbidez del efluente residual en un $81 \%$, mientras que T3 alcanzó una reducción promedio de $96 \%$. Con el tratamiento en serie (T3), se alcanzó un valor máximo inferior a 20UNT, para la turbidez y ligeramente superior a $100 \mathrm{mg} / \mathrm{L}$, para los sólidos totales. Con T2, el $100 \%$ de las concentraciones de turbiedad estuvieron por debajo de 80UNT, mientras que para T1, menos del 25\% de los datos, fue inferior a dicho valor. Para los sólidos totales, $100 \%$ de las concentraciones finales variaron en un rango de 200 a $300 \mathrm{mg} / \mathrm{L}$, en tratamientos con E. foetida, mientras que con $E$. crassipes, cerca del $75 \%$ de los datos fue inferior a este valor (Figura 2).

La $\mathrm{DBO}_{5}$ disminuyó en un porcentaje máximo de $91 \%$, al ser tratada en el sistema en serie (T3), seguido por T2, con $83,7 \%$. Más del $75 \%$ de las concentraciones finales estuvieron por debajo de $20 \mathrm{mg} / \mathrm{L}$ en T3, en contraste con T1; más del $75 \%$ de las concentraciones finales superaron dicho valor. Con T2, la degradación biológica de la materia orgánica reportó concentraciones por debajo de $40 \mathrm{mg} / \mathrm{L}$, en más del $75 \%$ de los casos.
En el caso de la DQO, T1, T2 y T3 reportaron concentraciones inferiores a $150 \mathrm{mg} / \mathrm{L}$, observándose una mejor agrupación de los datos con E. crassipes, lo cual, representa una mayor similitud entre los valores medidos. Para E. foetida, el $25 \%$ de los valores medidos estuvieron por debajo de $50 \mathrm{mg} / \mathrm{L}$, mientras que para los otros tratamientos, más del $50 \%$ de los valores se distribuyó en un rango de 50 a $100 \mathrm{mg} / \mathrm{L}$. Según Kadlec et al. (2000), la disminución en la concentración de DQO se debe, probablemente, al metabolismo de los macro y microorganismos heterótrofos aerobios y anaerobios, que utilizan los compuestos orgánicos del agua para la producción de biomasa y a las reacciones químicas de óxido reducción, a través de las cuales, se da dicho proceso.

Los resultados anteriores son corroborados por la prueba HDS de Tukey, determinando que existen grandes similitudes en la capacidad remoción de Turbiedad ( $p=0,931)$, entre $E$. foetida y $E$. crassipes; asimismo, se indica que las especies evaluadas, como agentes biorremediadores, tienen una capacidad aproximadamente igual, para disminuir los niveles de $\mathrm{DQO}$, presentes en el efluente residual doméstico, evaluado con un $\mathrm{p}$ valor de $0,934 \mathrm{y}$ valores promedio de concentración final de T1 $=74,854 \mathrm{mg} / \mathrm{L}, \mathrm{T} 2=81,334 \mathrm{mg} / \mathrm{L}$ y T3= 82,079mg/L.

Las eficiencias de materia orgánica medida, como DQO y $\mathrm{DBO}_{5}$, son inferiores a las publicados por Montoya et al. (2010), empleando humedales con macrófitas, cuyos porcentajes medios fueron de 97,31 y 100\%, con Canna limbata; 94,49 y 99,09\%, con Heliconia psittacorum; 97,39 y 99,45\%, con Phragmites sp, para cada parámetro, respectivamente. De la misma manera, la DBO5 se redujo, en porcentajes similares a los obtenidos por Rodríguez et al. (2010), utilizando humedales artificiales con E. crassipes, $86 \%$ y Lemna minor, $58 \%$, mientras que el tratamiento con E. crassipes, redujo en un menor porcentaje que Phragmites australis $(95,7 \%)$ y Typha dominguensis $(90,2 \%)$, las concentraciones de DQO, en el efluente residual doméstico (Romero et al. 2009). 

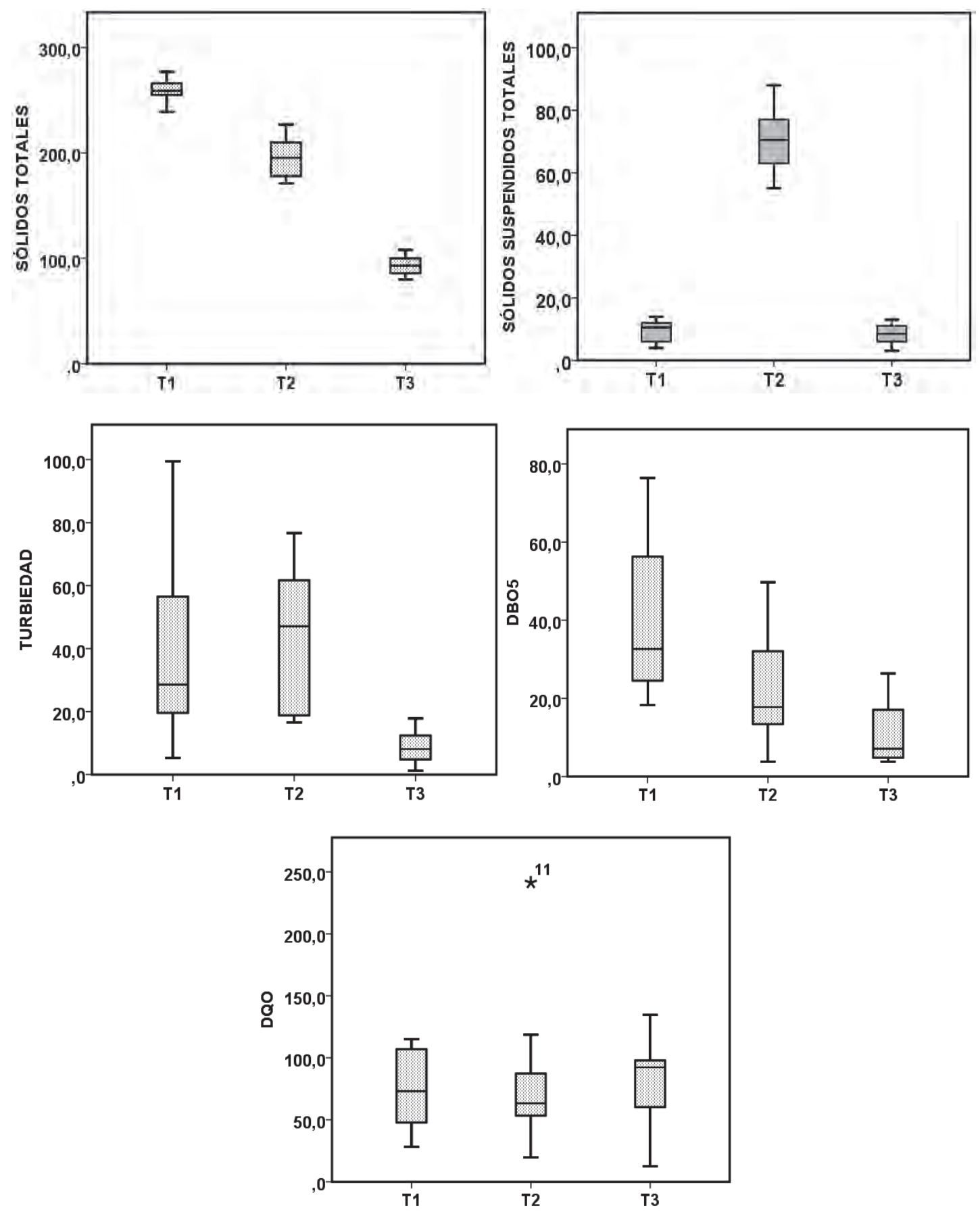

Figura 2. Concentraciones finales de Materia orgánica. Sólidos Totales, Sólidos suspendidos totales, Turbidez, DBO5 y DQO. T1) E. foetida; T2) E. crassipes; T3) E. foetida + E. crassipes.

En cuanto al comportamiento de los tratamientos frente a las concentraciones de nutrientes (Figura 3), T3 y T1 lograron las mejores remociones de nitrógeno total, con 79 y $73,6 \%$, respectivamente, con valores estadísticos de concentración $9,02 \mathrm{mg} / \mathrm{L}$ y $11,26 \mathrm{mg} / \mathrm{L}$, con una significancia $\mathrm{p}=0,599$. Con E. crassipes, la remoción de NT fue menor, lográndose una concentración estadística promedio de $21,14 \mathrm{mg} / \mathrm{L}$, para $p=1,000$. Esta reducción, se da a través de procesos, como su utilización por los organismos (Cervantes et al. 2000) como nutrimentos clave para su crecimiento y síntesis de proteínas, especialmente, en forma de nitrato (Romero et al. 2009), amonificación y nitrificación/denitrificación (Vymazal, 
2002). En el caso de T1, su eliminación en el efluente se da como consecuencia del consumo como fuente principal de alimento y su transformación en humus, por efecto de la acción enzimática del aparato digestivo de la lombriz (Díaz et al. 2008).

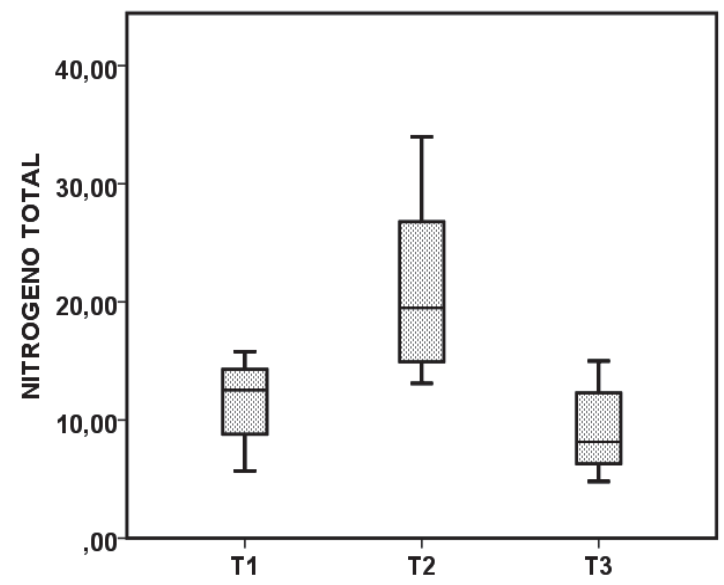

Los porcentajes de eliminación de NT logrados en los tres tratamiento aplicados son superiores a los reportados por Gutiérrez \& Peña (2011), empleando un humedal artificial de flujo subsuperficial con Heliconia psíttacorum, 56,7\% y menor que lo mencionado por Sawaittayothin \& Polprasert

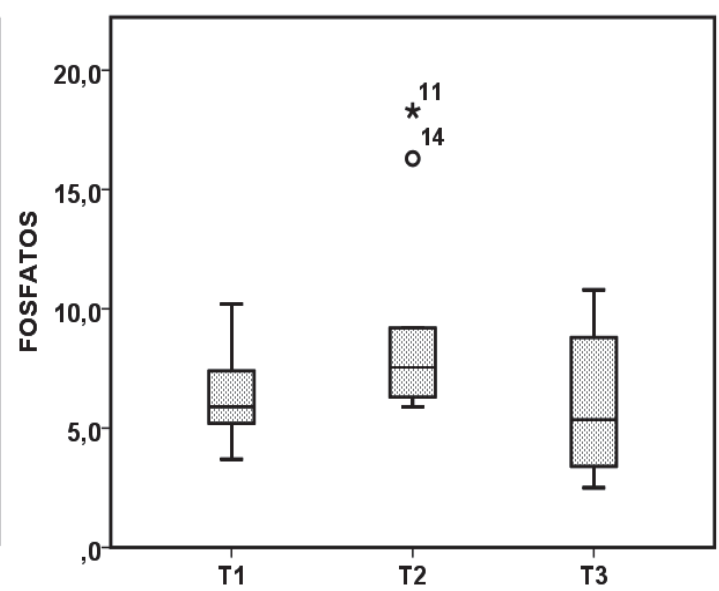

Figura 3. Concentraciones finales de Nutrientes: Nitrógeno total y fosfatos. T1) E. foetida; T2) E. crassipes; T3) E. foetida + E. crassipes.

(2007) con Typha angustifolia, para el mismo tipo de humedal, 96\%. Para el tratamiento combinado, las remociones de $\mathrm{DBO}_{5}$ y coliformes fecales son iguales a las de dicha planta, en el sistema en mención.

Las concentraciones de fosfatos guardan la relación $\mathrm{T} 3>\mathrm{T} 1>\mathrm{T} 2$, con una significancia $\mathrm{p}=0,080$, para eficiencias de $48,44,6$ y $20 \%$, respectivamente. Los resultados de T2, según lo citado por Brix (1987), se puede atribuir a la ausencia de un lecho de soporte, capaz de retener el fósforo, a través de mecanismos, como la precipitación y la adsorción; así como por un posible incremento en las concentraciones de fósforo inorgánico, como consecuencia de la actividad microbiana anaerobia (Hiley, 1995). Asimismo, estos resultados se pueden asociar al hecho que la eliminación de fósforo -en sistemas con plantas flotantes-, se debe a su consumo en el proceso de fotosíntesis, integrándose a su metabolismo de las macrófitas, bajo diversas formas iónicas, principalmente los fosfatos (Romero et al. 2009). Esta remoción es eficiente hasta que el medio se satura, lo cual, genera que los procesos sean limitados y se reduzca su asimilación (Karpiscak \& Foster, 2000).

Para T3, se obtuvo una concentración estadística de 5,99mg $\mathrm{PO}_{4}^{-3} / \mathrm{L}$, ligeramente menor a la lograda por E. foetida, $6,43 \mathrm{mg} \mathrm{PO}_{4}^{-3} / \mathrm{L}$. Esta diferencia, se puede asociar a un posible efecto sinérgico generado por las plantas y por los microorganismos asociados a sus raíces (Davies \& Cottigham,
1993), así como a su consumo como nutriente secundario, por parte de la lombriz roja.

Respecto a la remoción de indicadores de contaminación fecal (Figura 4), los tratamientos mostraron una eficiencia T3 $>$ T2 $>$ T1, lo cual, se observa claramente en la tabla 1 y se corrobora con los resultados obtenidos en la prueba HDS de Tukey. Igualmente, se determinó una mayor similitud en el comportamiento de los valores medios, obtenidos para la eliminación de $E$. coli, en T3 y T2 ( $\mathrm{p}=0,220)$.

Los resultados superan ligeramente las eficiencias en unidades anaerobias de tanque séptico y filtro anaerobio de flujo ascendente, referenciadas por Madera et al. (2005), para concentraciones iniciales de $1,8 \times 10^{7}$ UFC/100mL, que fluctuaron entre 74 y $96 \%$. Con el tratamiento en serie, $E$. foetida $+E$. crassipes, para coliformes totales y fecales, se alcanzaron valores de remoción del 99\%, similares a los logrados por Palacios (2006), empleando una planta de tipo convencional 99,9\%.

En el tratamiento con E. foetida, la remoción de patógenos se asocia a procesos físicos, como la filtración generada por el lecho o la sedimentación favorecida por la baja velocidad del flujo, así como por ingestión de estos por parte de las lombrices (Parra \& Chiang, 2014). En T2, la remoción se asocia a las bacterias adheridas a las raíces de la planta, las cuales, consumen como alimento o eliminan como consecuencia del 
efecto causado por algunas toxinas derivadas de su actividad metabólica, así como los protozoos, flagelados y ciliados, que actúan como depredadores (Ottová et al. 1997) y al efecto bactericida de las plantas utilizadas (Kuschk et al. 2003).
Basado en los resultados obtenidos, se concluye que la $E$. foetida y la $E$. crassipes son especies con gran capacidad para la reducción de la carga orgánica presente en efluentes residuales domésticos.
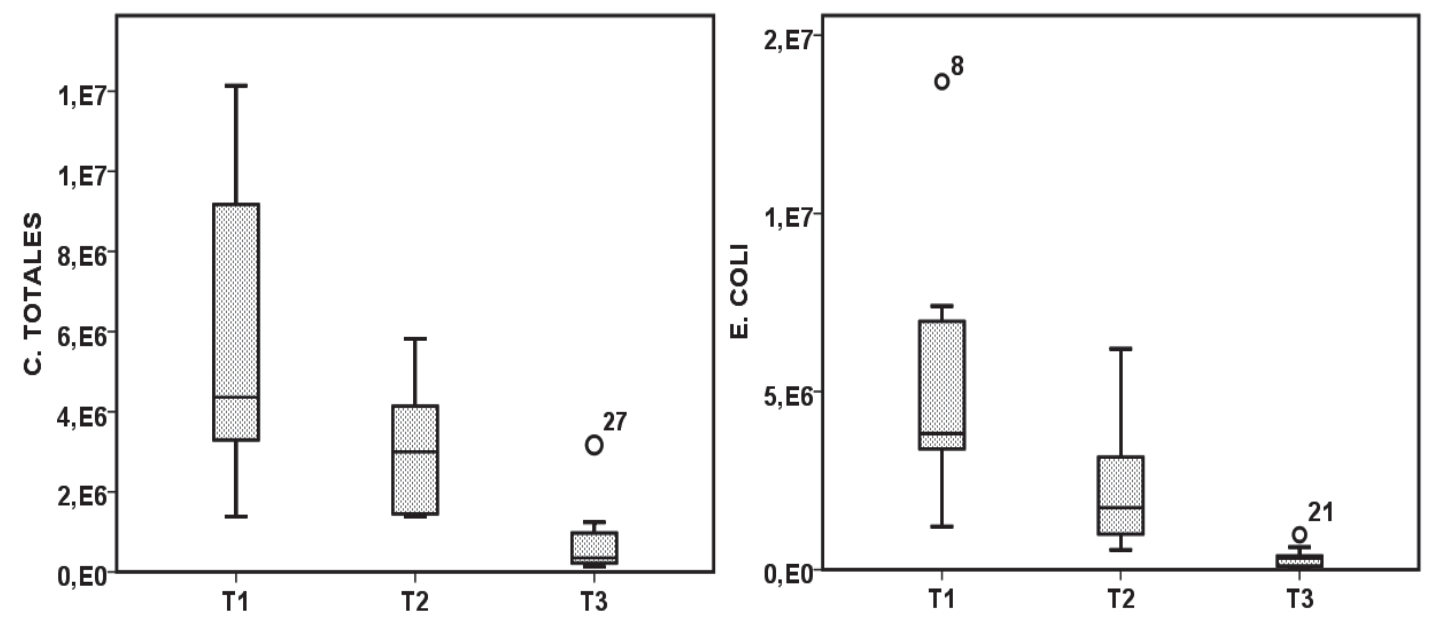

Figura 4. Concentraciones finales de Coliformes totales y E. coli. T1) E. foetida; T2) E. crassipes; T3) E. foetida + E. crassipes.

Al tratar el agua residual doméstica con un sistema en serie constituido por un biofiltro dinámico aerobio de flujo vertical con lechos de $E$. foetida más un sistema de flujo horizontal con E. crassipes, se incrementan las tasas de remoción de materia orgánica, nutrientes y patógenos, obteniéndose un efluente de mejor calidad, que el logrado al tratar el agua con cada especie por separado.

Agradecimientos: Los autores expresan su agradecimiento a la Universidad de La Guajira Sede Fonseca y a los auxiliares de investigación, José Alfredo Guerra y José Alfredo Gutiérrez, por su apoyo y compromiso en las fases de campo y laboratorio. Conflictos de intereses: El manuscrito fue preparado y revisado con la participación de los autores, quienes declaramos que no existe conflicto de intereses que ponga en riesgo la validez de los resultados presentados. Financiación: Esta investigación fue desarrollada con los recursos financieros proporcionados por los autores y auxiliares de investigación y con recursos técnicos aportados por la Universidad de La Guajira.

\section{BIBLIOGRAFÍA}

1. APHA-AWWA-WPCF. 2005. Standard Methods for Examination of Water and Wastewater. 21th Ed.

2. BRIX, H. 1987. Treatment of wastewater in the rhizosphere of wetlands plants the root zone method. Water Sci. Technol. (Inglaterra). 19:107-118.
3. CERVANTES, F.; PÉREZ, J.; GÓMEZ, J. 2000. Avances en la Eliminación Biológica del Nitrógeno de las Aguas Residuales Rev. Latinoam. Microbiología (México). 42:73-82.

4. CORREA, S.; GAMARRA, Y.; SALAZAR, A.; PITTA, N. 2015. Evaluación de la remoción de nitrógeno, fósforo y sulfuros en agua residual doméstica, utilizando Phragmites australis en Bioreactores. Inf. Tecnológ. 26(6): 89-98.

5. CORREA, G.; CUERVO, H.; MEJÍA, R.; AGUIRRE, N. 2012. Monitoreo del sistema de lagunas de estabilización del municipio de Santa Fé de Antioquia, Colombia. Producción + Limpia (Colombia). 7(2):3651.

6. DAVIES, T.; COTTINGHAM, P. 1993. Phosphorus removal from wastewater in a constructed wetland. En: Knight, L. (ed.) Lewis Publishers, Florida, 720p.

7. DÍAZ, D.; COVA, L.; CASTRO, A.; GARCÍA, D.; PEREA, F. 2008. Dinámica del crecimiento y producción de la lombriz roja californiana (Eisenia foetida Sav.) en cuatro sustratos a base de estiércol bovino. Agr. Andina. (Venezuela). 15:39-55.

8. GUTIÉRREZ, H.; PEÑA, M. 2011. Eliminación de nitrógeno en un humedal construido subsuperficial, 
plantado con Heliconia piittacorum. Tecnol. Cienc. Agua (México) 2(3):49-60.

9. HILEY, P. 1995. The reality of sewage treatment using wetlands. Water Sc. Technol. 32:329-337.

10. KADLEC, R.; KNIGHT, R.; VYMAZAL, J.; BRIX, H.; COOPER, P.; HABERL, R. 2000. Constructed wetlands for pollution control. Scientific and technical report No: 8. International Water Association (Londres). 156p.

11. KARPISCAK, W.; FOSTER, A. 2000. Nutrient and heavy metal uptake and storage in constructed wetland systems in Arizona wetland systems for water pollution control. Water Sci. Technol. 3:1271-1278.

12. KUSCHK, P.; WIEßNER, A.; KAPPELMEYER, U.; WEIßBRODT, E.; KÄSTNER, M.; STOTTMEISTER, U. 2003. Annual cycle of nitrogen removal in a pilotscale subsurface horizontal flow constructed wetlandin a moderate climate. Water Research. 37:42364242.

13. LUNA, V.; RAMÍREZ, H. 2004. Medios de soporte alternativos para la remoción de fosforo en humedales artificiales. Rev. Internal. Contam. Amb. (México). 20(1):31-38.

14. MADERA, C.; SILVA, J.; PEÑA, M. 2005. Sistemas combinados para el tratamiento de aguas residuales basados en tanque séptico - filtro anaerobio y humedales subsuperficiales. Ingen. Competitiv. (Colombia). 7(2):5-10.

15. MARÍN, J.; CHINGA, C.; VELÁSQUIEZ, A.; GONZÁLEZ, P.; ZAMBRANO, L. 2015. Tratamiento de aguas residuales de una industria procesadora de pescado en reactores anaeróbicos discontinuos. Cienc. Ing. Neogranada (Colombia). 25(1):27-42.

16. MARTELO, J.; LARA, J. 2012. Macrófitas flotantes en el tratamiento de aguas residuales: una revisión del estado del arte. Ingenier. Ciencia, (Colombia). 8(15):221-243.

17. METCALF \& EDDY. 1995. Ingeniería de Aguas Residuales. 2da Ed. McGraw Hill (España). 1485p.

18. MONTOYA, J.; CEBALLOS, L.; CASAS, J.; MORATÓ, J. 2010. Estudio comparativo de la remoción de materia orgánica en humedales construidos de flujo horizontal subsuperficial usando tres especies de macrófitas. Rev. EIA Escuela de Ingeniería de Antioquia (Colombia). 14:75-84.
19. OTTOVÁ, V.; BALCAROVA, J.; VYMAZAL, J. 1997. Microbial characteristics of constructed wetlands. Water Sc. Tech. 35(5):117-123.

20. PALACIOS, C. 2006. Tratamiento de aguas residuales domésticas en la estación científica Pedro Vicente Maldonado, Antártida- Verano Austral 2003- 2004. Rev. Tecnológ. ESPOL. 19(19):185-190.

21. PARRA, I.; CHIANG, G. 2014. Modelo integrado de un sistema de biodepuración en origen de aguas residuales domiciliarias. Una propuesta para comunidades periurbanas del centro sur de Chile. Gestión Ambiente (Colombia). 16(3):39-51.

22. PEÑA, E.; MADERA, C.; SÁNCHEZ, J.; MEDINA, J. 2013. Bioprospección de plantas nativas para su uso en procesos de biorremediación: caso Helicona psittacorum (heliconiacea). Rev. Acad. Colomb. Cienc. 27(145):469-481.

23. PEÑA, M.; VAN GINNEKEN, M.; MADERA, C. 2003. Humedales de flujo subsuperficial: una alternativa natural para el tratamiento de aguas residuales domésticas en zonas tropicales. Ingenier. Competitiv. 5(1):27-35.

24. PÉREZ, M.; DOMÍNGUEZ, E.; GONZALEZ, Y.; JIMÉNEZ, T. 2015. Diseño de un humedal subsuperficial vertical para la depuración de las aguas residuales de la Universidad Central "Marta Abreu" de Las Villas. Afinidad (España). 72(569):37-41.

25. RAMÓN, J.; LEÓN, J.; CASTILLO, N. 2015. Diseño de un sistema alternativo para el tratamiento de aguas residuales urbanas por medio de la técnica de lombrifiltros utilizando la especie Eisenia foetida. Mutis. (Colombia). 5(1):46-54.

26. ROMERO, M.; COLÍN, A.; SÁNCHEZ, E.; ORTIZ, M. 2009. Tratamiento de aguas residuales por un sistema piloto de humedales artificiales: evaluación de la remoción de la carga orgánica. Rev. Internal Contam. Amb. 25(3):157-167.

27. RODRÍGUEZ, J.; GÓMEZ, E.; GARAVITO, L.; LÓEZ, F. 2010. Estudio de comparación del tratamiento de aguas residuales domésticas utilizando lentejas y buchón de agua en humedales artificiales. Tecnol. Cienc. Agua (México). 1(1):59-68.

28. SALGADO, I.; DURÁN, C.; CRUZ, M.; CARBALLO, M.; MARTÍNEZ, A. 2012. Bacterias rizosféricas con potencialidades fisiológicas para eliminar materia 
orgánica de aguas residuales. Rev. Internal Contam. Amb. 28(1):17-26.

29. SAWAITTAYOTHIN, V.; POLPRASERT, C. 2007. Nitrogen mass balance and microbial analysis of constructed wetlands treating municipal landfill leachate. Biores. Tech. 98(3):565-570.

30. SEGHEZZO, L.; ZEEMAN, G.; VAN LIER, J.B.; HAMELERS, H.V.M.; LETTINGA, G. 1998. A review: the an- aerobic treatment of sewage in UASB and EGSB reactors. Biores. Technol. 65(3):175-190.

31. VYMAZAL, J. 2002. The use of sub-surface constructed wetlands for wastewater treatment in the Czech Republic: 10 years experience. Ecol. Engineer. 18:633646.

Recibido: Febrero 14 de 2016

Aceptado: Mayo 18 de 2016

Cómo citar:

Vizcaíno Mendoza, L.; Fuentes Molina, N. 2016. Efectos de Eisenia foetida y Eichhornia crassipes en la remoción de materia orgánica, nutrientes y coliformes en efluentes domésticos. Rev. U.D.C.A Act. \& Div. Cient. 19(1): 189-198. 